$57 \%$ überlebten, $21 \%$ mit Folgeschäden. Von 2010 bis 2017 lösten 11 Patienten den Notfall aus, wieder überlebten $57 \%$, keiner mit Folgeschäden, so Trappe. Der Schulungsaufwand war mit 1.500 potenziellen Ersthelfern wie Wachpersonal, Zöller und Flughafen-Angestellten beträchtlich.

Eine ähnliche Überlebensquote wurde in der Münchner U-Bahn erzielt. In den 37 U-Bahnhöfen sind 44 Defis platziert. Von 2001 bis 2011 gab es 17 Einsätze, 14 aufgrund eines plötzlichen Herztodes, davon 10 mit initialem Kammerflimmern. Von diesen Patienten verließen acht die Klinik ohne neurologische Schäden. Der Aufwand hierfür wird skeptisch gesehen: Beispiel Düsseldorfer Landtag. Für die ca. 100.000 Besucher sind 6 Defis installiert und 97 Landtags- mitarbeiter geschult worden. Das Experiment läuft seit über zehn Jahren, d.h. über eine Million Besucher weilten im Landtag, aber keiner fiel um, kein DefiEinsatz bis Ende September 2017.

Um einen kompletten Überblick über die inzwischen vorhandene Defi-Dichte und die Einsätze in ganz Deutschland zu bekommen, bräuchte man ein Register, dafür aber fehlt das Geld.

\section{Defis auch in der Wohnung?}

Ein weitergehender Vorschlag für die Verbreitung von Defis zielt auf Wohnungen von Herzpatienten. Eine entsprechende Empfehlung gab es in den USA. Immerhin weiß man, dass der plötzliche Herztod in $80 \%$ der Fälle im häuslichen Umfeld und nur in $20 \%$ im öffentlichen Raum auftritt.

\title{
Skandal oder Alarmismus - gefährden Blutdrucksenker unser Trinkwasser?
}

Es klingt nach Skandal: „Berliner Behörden schlagen Alarm - hohe Rückstände eines Blutdruckmittels gefährden TrinkwasserQualität", steht in einer DGK-Pressemitteilung. Vertreter der Berliner Wasserbetriebe (BWB) und des Landesamts für Gesundheit und Soziales (LAGeSo) haben angeblich auf den Herztagen mit der Mitteilung Aufsehen erregt, dass im Berliner Trinkwasser Rückstände von Sartanen, speziell Valsartan, messbar sind, die den Orientierungswert deutlich überschreiten und daher ein "humantoxikologisches Risiko" darstellen.

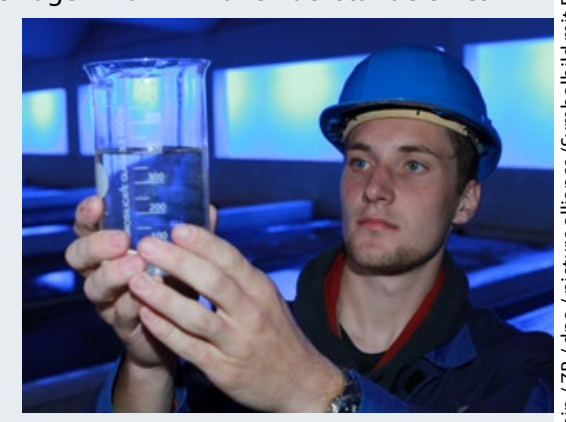

Abgesehen davon, dass die Experten von

BWB und LAGeSo im Tagungsprogramm nicht auftauchten, klingt die Meldung sehr nach Alarmismus. Wenn eine Gefahr für Wassertiere und Menschen drohte, hätte man eine ökotoxikologische Risikobewertung erwartet. Sie tut es aber (noch) nicht.

Obwohl zahlreiche Antihypertensiva ihre Spuren im Abwasser hinterlassen - jährlich sollen 15 Milliarden Tagesdosen in Deutschland verordnet werden - sei „Valsartan das einzige Antihypertensivum, das die Qualität der Trinkwasserressourcen in Deutschland gefährden kann." Als effiziente Maßnahme raten die Experten „eine Anpassung der Verordnungspraxis durch behandelnde Ärzte." Allein die Substitution von Valsartan durch Candesartan helfe, da es „unter den Sartanen die geringste Wirkstoffkonzentration je Tagesdosis aufweist (Faktor 10-18) und zum gleichen Preis erhältlich ist." Auf der Webseite der BWB findet sich im Frage-Antwort-Kapitel im übrigen folgende Passage:

"Jeder Einzelne von uns kann einen Teil zur Reduzierung von Spurenstoffen beitragen, etwa durch die gezielte und sparsame Einnahme von Medikamenten. Fragen Sie Ihren Arzt, ob er etwas über die Umweltwirksamkeit des verordneten Medikaments weiß und ob es eine ebenso wirksame, aber für die Umwelt und den Wasserkreislauf weniger schädliche Alternative gibt. Denn es gibt Arzneimittel, die im Klärwerk oder spätestens auf dem Weg ins Grundwasser komplett abgebaut werden. Wichtig ist, nicht benutzte oder abgelaufene Arzneimittel auf keinen Fall in die Toilette zu werfen, sondern sie umweltgerecht über die Schadstoffsammelstellen der BSR (www.bsr.de) zu entsorgen."

Vielleicht fühlen sich ja die Zulassungsbehörden angesprochen und fordern künftig eine ökotoxikologische Risikobewertung.

Eine großangelegte Vergleichstudie zwischen CRP mit und ohne Defi in Wohnungen von 7.001 Risikopatienten mit Vorderwandinfarkt, die keine Kandidaten für einen implantierbaren Kardioverter-Defibrillator waren, überprüfte dieses Konzept (Bardy GH et al. N Engl J Med. 2008;358:1793). Entgegen der Erwartungen brachte der Defi in der Wohnung keinen Überlebensvorteil.

In Deutschland äußerte sich Prof. Hans-Jürgen Becker von der Deutschen Herzstiftung zu der Idee, den Defi etwa der Hausapotheke anzuschließen: „Ob das eine sinnvolle Anschaffung darstellt, ist völlig offen. Unklar ist etwa, ob Defis bei drohendem plötzlichen Herztod in der privaten Wohnung nicht eher dazu beitragen, dass es zu Verzögerungen bei der Notarztalarmierung kommt oder die Herzdruckmassage und Beatmung vernachlässigt werden, die bei solch einem Notfall ebenfalls enorm wichtig sind." Diese private Investition scheint sich also nicht zu lohnen.

Vorgeschlagen wurde zudem, Schulen mit Defis auszustatten. Auch hier kann nicht übersehen werden, dass die Inzidenz von Herzstillständen in Schulen eine fast zu vernachlässigende Größe ist.

Dass die Verfügbarkeit von Defis bisweilen jenseits vernünftiger Argumenten eingefordert wird, kann die Deutsche Herzstiftung bestätigen, die viel Geld in den Ausbau des Defi-Netzes gesteckt hat. Aber wenn dann Eltern anfragen, ob der Kindergarten mit ihren Sprösslingen einen lebensrettenden Defi bekommen könnte, wird klar, dass das Konzept missverstanden wird.

Auch Trappe lehnt es ab, Defis aus Unkenntnis sowie aus Image- oder PRGründen vorzuhalten, vor allem dann, wenn es eine isolierte Aktion bleibt, ohne begleitende Schulungsanstrengungen. Vermutlich sollte man die Vorzüge des Defis nur noch in Verbindung mit der CPR propagieren.

Zusammenfassend ist es für Trappe unbestritten, dass der Defi bei Patienten mit Kammertachykardie bzw. -flimmern die Überlebensrate verbessert. Die Anzahl der Einsätze ist aber wesentlich geringer als erwartet. Die Wahrheit ist, dass „viele Defis und viele geschulte Ersthelfer nötig sind, um nur ein Leben zu retten.“ Dr. med. Jochen Aumiller 